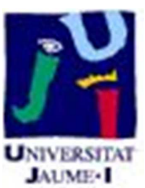

Título artículo / Títol article: Measures of Similarity between Qualitative Descriptions of Shape, Colour and Size Applied to Mosaic Assembling

Autores / Autors

Falomir Llansola, Zoe ; Museros Cabedo, Lledó ;

González Abril, Luís ; Velasco, Francisco

Revista:

Journal of Visual Communication and Image

Representation

Versión / Versió:

Pre-print

Cita bibliográfica / Cita bibliogràfica (ISO 690):
FALOMIR, Zoe, et al. Measures of similarity between qualitative descriptions of shape, colour and size applied to mosaic assembling. Journal of Visual Communication and Image Representation, 2013, vol. 24, no 3, p. 388-396. 


\title{
Measures of Similarity between Qualitative Descriptions of Shape, Colour and Size Applied to Mosaic Assembling
}

\author{
Zoe Falomir $^{\mathrm{a}, \mathrm{b}}$, Lledó Museros ${ }^{\mathrm{b}}$, Luis Gonzalez-Abril $^{\mathrm{c}}$, Francisco Velasco $^{\mathrm{c}}$ \\ ${ }^{a}$ Cognitive Systems (CoSy), FB3 - Informatics, Universität Bremen, Enrique-Schmidt-Str. 5, 28359 Bremen, Germany \\ ${ }^{b}$ Engineering and Computer Science Department, Universitat Jaume I, Escola de Tecnologia i Ciéncies Experimentals, \\ Av. Vicent Sos Baynat s/n, E-12071, Castelló, Spain \\ ${ }^{c}$ Applied Economics I Department, Universidad de Sevilla, Facultad de Ciencias Económicas, Avda. Ramon y Cajal, 1, \\ E-41018, Sevilla, Spain
}

\begin{abstract}
A computational approach for obtaining a similarity measure between qualitative descriptions of shape, colour and size of objects within digital images is presented. According to the definition of the qualitative features, the similarity values determined are based on conceptual neighbourhood diagrams or interval distances. An approximate matching algorithm between object descriptions is defined and applied to tile mosaic assembling and results of previous approaches are improved. Keywords: Qualitative Shape, Qualitative Colour, Qualitative Size, Conceptual Neighbourhood Diagram, Interval Distance, Similarity Measure, Mosaic Assembling
\end{abstract}

\section{Introduction}

Mosaicing is the art of creating images with an assemblage of small pieces. These small pieces can be: (i) multiple, overlapping images that stitch together in order to produce one large, high resolution composite or panorama; or (ii) small coloured glass, stones, marbles and/or ceramic tiles, known as tesserae (diminutive tessellae), which are assembled to create a predefined design.

In the literature there are research works that automate mosaicing for the creation of high resolution composites or panoramas. For example, Chailloux et al. [1] defined an automatic intensity-based registration algorithm which used overlapping sonar images to build a full seafloor

Email addresses: zfalomir@\{informatik.uni-bremen.de, uji.es\} (Zoe Falomir), museros@uji.es (Lledó Museros), luisgon@us.es (Luis Gonzalez-Abril), velasco@us.es (Francisco Velasco)

Preprint submitted to Journal of Visual Communication and Image Representation 
panorama; or the work by Liang et al. [2] which produced a high resolution and accurate full page mosaic from small image patches of a document.

Mosaics as decorative art forms built by tesserae are the focus of this paper. The mosaic industry is very important for the economy of the countries of southern Europe and north Africa. Despite its relevance, mosaic assembly is a task carried out mainly manually nowadays. Only some automatic systems for assembling tesserae are partially introduced in industrial coverings sectors (i.e., Artaic ${ }^{1}$, Top Hat Tile ${ }^{2}$, Tilemyzer ${ }^{3}$, and Rhinoceros [3]). These automatic systems assemble mosaics composed only by quadrilateral tesserae.

In the literature, some assembling automations can be found which obtained successful results using squared/rectangular tesserae, with same or different colours, and with mainly the same size $[4,5,6,7]$. Navon [4] implemented a camera-based application for quality control of a floor tiling robot for assembling squared large prefabricated components into walls and floors. Oral and Erzincanli [5] developed a software for tiling mosaics automatically by picking up the marble pieces from the supply stations and placing them according to their coordinates. The authors formed squared and quadrangled shaped mosaic configurations with pieces of fixed size using a computer controlled SCARA robot. Kaya et al. [6] developed an image processing software for tiling glass mosaics by a Ultimate Puma 500 industrial robot which could use pieces of different colours with the same squared shapes. Cayiroglu and Demir [7] developed a system for tiling mosaics made by squared shaped tiles of fixed size which was fully automated: starting from a digital photo, through to the tiling of mosaics, up to the packaging of panels in the production, performed without any manual intervention in a continuous flow.

However, obtaining automatic systems that assemble mosaics in decorative strips or borders using pieces with more sophisticated shapes such as sharp angles, curves and/or concavities is still a challenge. There are some works in the literature approaching the solution which can be classified into: (i) systems using a numerical description of the design [8]; and (ii) systems using a qualitative description of the mosaic $[9,10,11,12,13,14]$.

Oral and Inal [8] developed an algorithm for marble mosaic tiling using the geometrical data of the pieces obtained from 2D CAD software and the 2D CAD mosaic pattern. These numerical data were interpreted by control software that enabled a Cartesian robot to assemble mosaic

\footnotetext{
${ }^{1}$ Artaic: http: //www .artaic. com/ (Access date: Oct 2012)

${ }^{2}$ Top Hat Tile: http : / /www . tophattile.com/ (Access date: Oct 2012)

${ }^{3}$ Tilemyzer: http : / /www.tilemyzer. com (Access date: Oct 2012)
} 
pieces of different sizes and shapes according to patterns on hand.

An approach for assembling mosaics by exact matching of pieces described qualitatively and RGB colour values was presented by Museros et al. [9, 10, 11, 12]. However, as some qualitative terms used in a description of shape are related to an interval of values, it can happen that two very close numerical values located on the limit of those intervals are assigned different qualitative terms. Exact matching discards two very close shapes which obtains different but very similar qualitative descriptions. Therefore, for avoiding this problem and accelerating the mosaic assembling process, a measure of similarity between qualitative descriptions of objects is required. And as object recognition involves identifying the shape, colour and size of the object, three similarity measures regarding those features are needed.

Object shape description and comparison has been widely studied in literature. Approaches that calculate a similarity measure between quantitative shape descriptions are classified as using: (i) points of high curvature [15] or landmark points [16] in the shape contour; (ii) the matching of total or partial contours [17]; (iii) shape feature descriptor vectors [18]; (iv) shock graphs/trees $[19,20]$, etc. Other approaches for qualitative shape comparison can be classified as calculating a similarity measure: (i) between qualitative descriptions based on bipartite arrangements for matching partial or total contours [21]; (ii) based on conceptual neighbourhood diagrams for comparing qualitative shape descriptions [22]; (iii) based on matrices of qualitative concepts for comparing polygons [23]; (iv) based on scope histograms for comparing polygons described qualitatively [24].

Moreover, in literature, colour similarity measures were defined mainly on numerical colour spaces, such as: (i) Euclidean distance for cubic spaces as RGB or CIE Lab and for cylindric spaces as $\mathrm{L} * \mathrm{C} * \mathrm{H}$ [25]; (ii) a cylindric distance for cylindric and conic spaces like HSL, HSV and $\mathrm{L} * \mathrm{C} * \mathrm{H}[26]$; (iii) the Fuzzy C-Means for defining similarity measures for comparing fuzzy colour categories based on Musell colour space [27]. There are less studies in literature that provide a similarity measure between qualitative colours using: (i) surveys to people asking the relation between colour names [28] and conceptual neighbourhood diagrams [13, 14].

In this paper, an approach that describe qualitatively the shape, colour and size of real objects contained within a digital image and that calculates a similarity measure between those descriptions is presented and applied to mosaic assembling. This approach differs from the previous works by Museros et al. $[9,10,11,12]$ in the following points: (1) the current qualitative des- 
cription of shape introduces a new qualitative concept for describing the edge connection and it also presents a higher granularity for the qualitative concepts angle, compared length and type of curvature, allowing to differentiate between more shapes [29]; (2) the colour of each object is described qualitatively instead of using its RGB coordinates, which is a more cognitive method; (3) the size of the objects is described qualitatively comparing it to the rest of the objects composing the mosaic, which does not limit our approach to the predefined pieces that compose the mosaic; and (4) the matching algorithm is similarity-based, instead of using all-or-nothing matching of qualitative features or RGB values, which speeds up the process.

A pilot study for solving the problem of mosaic assembling by approximate matching was presented by Falomir et al. [13, 14] using only shape and colour similarity values calculated using conceptual neighbourhood diagrams (CNDs). However, a more effective approach is formulated here by introducing a new qualitative size description and also by combining both CNDs and interval distances for obtaining a similarity between qualitative shapes, and using interval distances for measuring the similarity between qualitative colours and also between qualitative sizes.

The rest of this paper is structured as follows. The qualitative description of the shape, colour and size of any object is presented in Section 2. Similarity measures between qualitative descriptions of shape, colour and size are given in Section 3. In Section 4, the matching algorithm according to the application is presented and the results of the experimentation are shown. In Section 5 a discussion is provided regarding further applications, the results obtained here compared to those obtained in the literature, and the economic justification of the presented approach. Finally, conclusions are drawn in Section 6.

\section{Qualitative Description of a Tesserae}

This approach automatically extracts the closed boundary of the objects within any digital image (for further details see Falomir et al. [30]) and describe their shape as explained in Section 2.1. It also calculates the centroid of each object and obtains its qualitative colour (Section 2.2) and its area for classifying it according to its qualitative size (Section 2.3). Finally, a complete qualitative description of the object is given (Section 2.4).

\subsection{Qualitative Shape Description (QSD)}

This approach analyses the slope of the pixels within the object boundary and extracts the relevant points of its shape. Each of these relevant points $\left(\left\{P_{0}, P_{1}, \ldots . P_{N}\right\}\right)$ is described by a set of four 
features $<\mathrm{EC}_{P}, \mathrm{~A}_{P}$ or $\mathrm{TC}_{P}, \mathrm{~L}_{P}, \mathrm{C}_{P}>$, which are defined below.

The first feature is the Edge Connection (EC) occurring at $P$. This feature is described by the following tags:

- line-line, if the point $P$ connects two straight lines;

- line-curve, if $P$ connects a line and a curve;

- curve-line, if $P$ connects a curve and a line;

- curve-curve, if $P$ connects two curves; or

- curvature-point, if $P$ is a point of curvature of a curve.

If $\mathbf{E C}$ is a line-line, line-curve, curve-line or curve-curve, the second feature to consider is the Angle (A) at $P$ that is a quantitative feature discretized using the Angle Reference System or $\mathrm{ARS}=\left\{{ }^{\circ}, \mathrm{A}_{L A B}, \mathrm{~A}_{I N T}\right\}$ where,$^{\circ}$ (degrees) indicates the unit of measurement; $\mathrm{A}_{L A B}$ refers to the set of labels defined; and $\mathrm{A}_{I N T}$ refers to the values of degrees $\left(^{\circ}\right)$ related to each label. In our approach:

$\mathrm{A}_{L A B}=\{$ very_acute, acute, right, obtuse, very_obtuse $\}$

$\mathrm{A}_{I N T}=\{(0,40],(40,85],(85,95],(95,140],(140,180]\}$

If EC is a curvature-point, the second feature is the Type of Curvature (TC) at $P$ defined by the Type of Curvature Reference System or TCRS $=\left\{{ }^{\circ}, \mathrm{TC}_{L A B}, \mathrm{TC}_{I N T}\right\}$, where ${ }^{\circ}$ refers to the amplitude in degrees of the angle of curvature at $P ; \mathrm{TC}_{L A B}$ refers to the set of labels defined; and $\mathrm{TC}_{I N T}$ refers to the values in degrees related to each label. In our approach:

$\mathrm{TC}_{L A B}=\{$ very_acute, acute, semicircular,plane,very plane $\}$

$\mathrm{TC}_{I N T}=\{(0,40],(40,85],(85,95],(95,140],(140,180]\}$

The third feature considered is the compared length $(\mathbf{L})$ defined by the Length Reference System or LRS $=\left\{\mathrm{UL}, \mathrm{L}_{L A B}, \mathrm{~L}_{I N T}\right\}$, where UL or Unit of compared Length refers to the relation between the length of the first edge and the length of the second edge connected by $P ; L_{L A B}$ refers to the set of labels for compared length; and $\mathrm{L}_{I N T}$ refers to the values of UL related to each label. In our approach:

$\mathrm{L}_{L A B}=\{$ much_shorter $(m s h)$, half 」ength (hl), a bit_shorter (absh), similar Jength (sl), a bit longer (abl), double length $(\mathrm{dl})$, much longer $(\mathrm{ml})\}$

$\mathrm{L}_{I N T}=\{(0,0.4],(0.4,0.6],(0.6,0.9],(0.9,1.1],(1.1,1.9],(1.9,2.1],(2.1, \infty)\}$ 
The last feature to be considered is the Convexity (C) at point $P$. If point $P_{j}$ is on the left of the segment defined by $\mathrm{P}_{j-1}$ and $\mathrm{P}_{j+1}$, then $P_{j}$ is convex; otherwise $P_{j}$ is concave.

Thus, the complete shape of an object is described as a set of qualitative descriptions of relevant points as ${ }^{4}$ :

$$
\left[\left[\mathrm{EC}_{1}, \mathrm{~A}_{1} \mid \mathrm{TC}_{1}, \mathrm{~L}_{1}, \mathrm{C}_{1}\right], \ldots,\left[\mathrm{EC}_{n}, \mathrm{~A}_{n} \mid \mathrm{TC}_{n}, \mathrm{~L}_{n}, \mathrm{C}_{n}\right]\right]
$$

where $\mathbf{n}$ is the total number of relevant points of the object.

The intervals of values that define the qualitative tags representing the features A, TC and $\mathrm{L}\left(\mathrm{A}_{I N T}, \mathrm{TC}_{I N T}\right.$ and $\mathrm{L}_{I N T}$, respectively) can be generalized. However, here they have been calibrated according to the application and system given in this paper.

\subsection{Qualitative Colour Description (QCD)}

The approach presented translates the Red, Green and Blue (RGB) colour channels into Hue, Saturation and Lightness (HSL) coordinates, which are more suitable for dividing into intervals of values corresponding to colour names.

From the HSL colour coordinates obtained, a reference system for qualitative colour description is defined as: $\mathrm{QCRS}=\left\{\mathrm{UH}, \mathrm{US}, \mathrm{UL}, \mathrm{QC}_{L A B 1 . .5}, \mathrm{QC}_{I N T 1 . .5}\right\}$ where $\mathrm{UH}$ is the Unit of Hue; US is the Unit of Saturation; UL is the Unit of Lightness; $\mathrm{QC}_{L A B 1 . .5}$ refers to the qualitative labels related to colour; and $\mathrm{QC}_{I N T 1.5}$ refers to the intervals of HSL colour coordinates associated with each colour label. For our approach, the $\mathrm{QC}_{L A B}$ and $\mathrm{QC}_{I N T}$ are the following:

$\mathrm{QC}_{L A B_{1}}=\{$ black, dark_grey, grey, light_grey, white $\}$

$\mathrm{QC}_{I N T_{1}}=\{[0,20),[20,30),[30,40),[40,80),[80,100) \in \mathrm{UL} /[0,360] \in \mathrm{UH} \wedge[0,20] \in \mathrm{US}\}$

$\mathrm{QC}_{L A B_{2}}=\{$ red, yellow, green, turquoise, blue, purple, pink $\}$

$\mathrm{QC}_{I N T_{2}}=\{(335,360] \wedge[0,40],(40,80],(80,160],(160,200],(200,260],(260,297],(297,335] \in \mathrm{UH} /$

$(50,100] \in \mathrm{US} \wedge(40,55] \in \mathrm{UL}\}$

$\mathrm{QC}_{L A B_{3}}=\left\{\right.$ pale $\left.-+\mathrm{QC}_{L A B_{2}}\right\}$

$\mathrm{QC}_{I N T_{3}}=\{(335,360] \wedge[0,40],(40,80],(80,160],(160,200],(200,260],(260,297],(297,335] \in \mathrm{UH} /$

$(20,50] \in \mathrm{US} \wedge(40,55] \in \mathrm{UL}\}$

$\mathrm{QC}_{L A B_{4}}=\left\{\right.$ light $\left.+\mathrm{QC}_{L A B_{2}}\right\}$

\footnotetext{
${ }^{4} \mathrm{~A}_{i} \mid \mathrm{TC}_{i}$ denotes the angle or the type of curvature that occurs at the point $P_{i}$.
} 
$\mathrm{QC}_{I N T_{4}}=\{(335,360] \wedge[0,40],(40,80],(80,160],(160,200],(200,260],(260,297],(297,335] \in \mathrm{UH} /$

$(50,100] \in \mathrm{US} \wedge(55,100] \in \mathrm{UL}\}$

$\mathrm{QC}_{L A B_{5}}=\left\{\right.$ dark $\left._{-}+\mathrm{QC}_{L A B_{2}}\right\}$

$\mathrm{QC}_{I N T_{5}}=\{(335,360] \wedge[0,40],(40,80],(80,160],(160,200],(200,260],(260,297],(297,335] \in \mathrm{UH} /$

$(50,100] \in \mathrm{US} \wedge(20,40] \in \mathrm{UL}\}$

The saturation coordinate (US) determines if the colour corresponds to the grey or to the rainbow scale: $\mathrm{QC}_{L A B 1}$ and $\mathrm{QC}_{L A B 2}$, respectively, in this QCRS. This coordinate also determines the intensity of the colour (pale or strong). The colours in the rainbow scale are considered as the strong ones, while the pale colours are given an explicit name in $\mathrm{QC}_{L A B 3}$. The hue coordinate (UH) determines the division into colour names inside each scale. The lightness coordinate (UL) determines the luminosity of the colour: dark and light colours are given an explicit name in $\mathrm{QC}_{L A B 4}$ and $\mathrm{QC}_{L A B 5}$, respectively. Finally, once more, note that the $\mathrm{QC}_{I N T 1 . .5}$ were calibrated according to our vision system.

\subsection{Qualitative Size Description (QZD)}

The qualitative size of an object (i.e. small, medium, big) is calculated geometrically using the coordinates of the relevant points of the shape boundary [31]. The sizes of all the objects belonging to the same family or set are considered as the reference. The cumulative algorithms used [32], given the number of intervals to reach and a set of object sizes, can determine the limits of each interval by minimising the mean of the standard deviation inside each interval. This leads to find values maximising the representativeness of each tag within its interval. From the interval classes obtained by these algorithms, a reference system for qualitative size or SRS

is defined as $\mathrm{SRS}=\left\{\mathrm{US}, \mathrm{S}_{L A B}, \mathrm{~S}_{I N T}\right\}$, where US refers to the size of the object; $\mathrm{S}_{L A B}$ refers to the set labels that represent size; and $S_{I N T}$ refers to the intervals associated to each label. In our approach:

$\mathrm{S}_{L A B}=\{$ very_small, small, medium, large, very_large $\}$

$\mathrm{S}_{I N T}=\{(10213,16302],(16302,28481],(28481,34571],(34571,58929],(58929,65018]\}$

Note that the $\mathrm{S}_{I N T}$ values are calculated for our application. For a different experimentation different limits can be obtained.

\subsection{Qualitative Object Description (QOD)}

The complete qualitative description of an object is obtained as a set of variables as: 
[QCD, QZD, QSD] that is,

$$
\text { [QCD, QZD, [EC } \left.\left.1, \mathrm{~A}_{1} \mid \mathrm{TC}_{1}, \mathrm{~L}_{1}, \mathrm{C}_{1}\right], \ldots,\left[\mathrm{EC}_{n}, \mathrm{~A}_{n} \mid \mathrm{TC}_{n}, \mathrm{~L}_{n}, \mathrm{C}_{n}\right]\right]
$$

where $\mathbf{n}$ is the total number of relevant points of the object. An example is shown in Table 1.

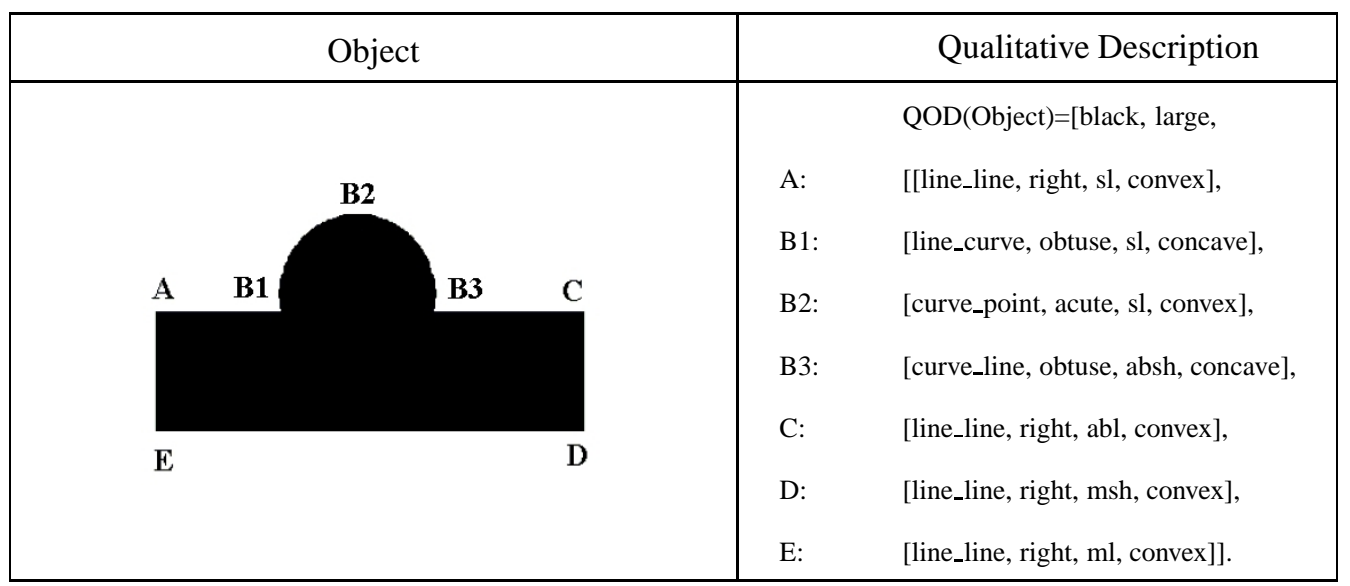

Table 1: Qualitative description of an object.

Finally, note that, an object or hole placed inside another object is described by its boundary in the same way as its container, and its location inside the container is indicated if necessary according to the application. For further details, please see Falomir et al. [30].

\section{Measures of Similarity between Tesserae}

For comparing two object descriptions, it is necessary to define similarity values between their QSDs, QCDs and QZDs.

\subsection{Similarity of $Q S D s$}

For obtaining a similarity measure between QSDs, three previous definitions are needed: (1) Similarity between qualitative tags related to the same feature of shape; (2) Similarity between relevant points; and (3) Similarity between QSDs by correspondence of their relevant points (Ps).

\subsubsection{Similarity between Qualitative Features}

This approach uses two measures of similarity between qualitative terms: (i) based on conceptual neighbourhood diagrams, for pure qualitative terms: EC and C; and (ii) based on interval 


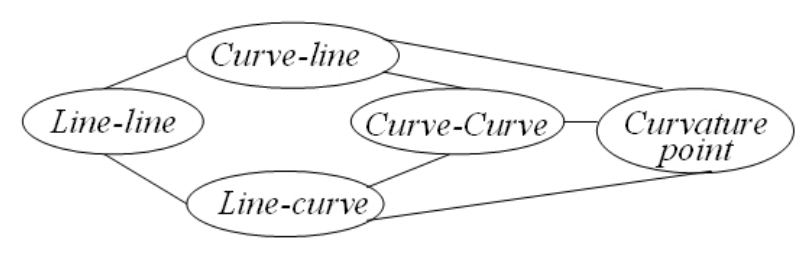

Figure 1: CND for feature Edge Connection (EC).

\begin{tabular}{|c|c|c|c|c|c|}
\hline EC & line line & line curve & curve line & curve curve & curvature point \\
\hline line line & 0 & 1 & 1 & 2 & 2 \\
\hline line curve & 1 & 0 & 2 & 1 & 1 \\
\hline curve line & 1 & 2 & 0 & 1 & 1 \\
\hline curve curve & 2 & 1 & 1 & 1 & 0 \\
\hline curvature point & 2 & 1 & 1 & 1 & 0 \\
\hline
\end{tabular}

Table 2: Dissimilarity matrix for EC using a CND.

distances for the qualitative terms defined as a discretization of a value on a reference system: A, TC and L.

Two qualitative terms are conceptual neighbours if 'one can be directly transformed into another by continuous deformation' [33]. For example, any connection involving a curve can evolve directly to a curvature-point but not to a line-line segment, because first it has to evolve to a curve-line or line-curve connection.

In general, Conceptual Neighbourhood Diagrams or CNDs can be described as diagrams or graphs containing: (i) nodes that map to a set of individual relations defined on regions or intervals and (ii) paths or edges connecting pairs of adjacent nodes that map to continuous transformations between them.

Figure 1 shows the CND proposed for the feature EC (for feature $\mathrm{C}$ is trivial) and Table 2 shows the dissimilarity between two labels calculated from the CND as the minimal path between them. The same is done for the feature $\mathrm{C}$.

The qualitative names for the features of shape $\mathrm{A}, \mathrm{TC}$ and $\mathrm{L}$ are defined from intervals of values in their Reference Systems. Hence, interval distances can be used to measure the dissimilarity between them. 
Let us introduce the concept of interval distance. Given an open interval (analogously for another kind of interval) of finite dimension, there are two main ways to represent it: from the extreme points as $(a, b)$ or as an open ball $\mathrm{B}_{r}(\mathrm{c})$ (Borelian notation) where $c=(a+b) / 2$ (centre) and $r=(b-a) / 2$ (radius). Given two intervals, $I_{1}=\left(a_{1}, b_{1}\right)=B_{r 1}\left(c_{1}\right)$ and $I_{2}=\left(a_{2}, b_{2}\right)=B_{r 2}\left(c_{2}\right)$, a family of distances between intervals is defined by Gonzalez-Abril et al. [34], as follows:

$$
d^{2}\left(I_{1}, I_{2}\right)=(\Delta c \Delta r) A\left(\begin{array}{c}
\Delta c \\
\Delta r
\end{array}\right)
$$

where $\Delta c=c_{2}-c_{1}, \Delta r=r_{2}-r_{1}$ and $A$ is a symmetrical $2 \times 2$ matrix of weights that must be a positive definite matrix. From the $A$ matrix, the weights given to the position of the intervals and to the radius can be controlled. In this paper, the identity matrix is used that provides the next distance:

$$
d\left(I_{1}, I_{2}\right)=\sqrt{\Delta^{2} c+\Delta^{2} r}=\sqrt{\left(a_{2}-a_{1}\right)^{2}+\left(b_{2}-b_{1}\right)^{2}}
$$

Hence, applying (1) to $\mathrm{A}_{I N T}, \mathrm{TC}_{I N T}$ and $\mathrm{L}_{I N T}$, matrices of dissimilarities between the qualitative names in $\mathrm{A}_{L A B}, \mathrm{TC}_{L A B}$ and $\mathrm{L}_{L A B}$ can be obtained. Note that the intervals in $\mathrm{A}_{I N T}$ and $\mathrm{TC}_{I N T}$ have different amplitudes and that interval distances take into account the amplitude of the compared intervals to calculate the dissimilarity between two qualitative names.

\subsubsection{Similarity between Relevant Points (Ps)}

As the qualitative shape of an object is described by its relevant points, a similarity between them is needed. Hence, given two relevant points, denoted by $P_{A}$ and $P_{B}$, belonging to the shapes of the objects A and B respectively, a similarity between them, denoted by $\operatorname{Sim}\left(P_{A}, P_{B}\right)$, is defined as:

$$
\operatorname{Sim}\left(P_{A}, P_{B}\right)=1-\sum_{i \in\{E C, A \vee T C, C, L\}} w_{i} \frac{d s(i)}{D s(i)},
$$

where $d s($ feature $)$ and $D s($ feature $)$ denote the dissimilarity between relevant points and the maximum dissimilarity respectively respect to the feature obtained from the dissimilarity matrix. Hence, by dividing $d s$ (feature) and Ds(feature) the proportion of dissimilarity related to feature of $\mathrm{P}_{A}$ and $\mathrm{P}_{B}$ is obtained, which is between 0 and 1 . Moreover, the parameter $w_{\text {feature }}$ is the weight assigned to this feature, and it holds that $w_{E C}+w_{A}+w_{L}+w_{C}=1, w_{A}=w_{T C}$ and $w_{\text {feature }} \geq 0$ for each feature.

In this paper, with the aim of giving the same importance to all features in (2), all the weights

have the same value: $\frac{1}{4}$. Clearly, these weights can be tuned if a researcher needs to give more 
importance to one feature over the others. Furthermore, in (2) it is subtracted from 1 with the aim of providing a similarity instead of a dissimilarity.

For each $P_{A}$ and $P_{B}$, it is straightforward to prove that $0 \leq \operatorname{Sim}\left(P_{A}, P_{B}\right) \leq 1$ and that this is a symmetrical relation. Furthermore, $\operatorname{Sim}\left(P_{A}, P_{B}\right)=0$ means that both relevant points are as different as possible, and $\operatorname{Sim}\left(P_{A}, P_{B}\right)=1$ means that both relevant points are the same.

\subsubsection{Similarity of $Q S D$ s by Correspondence of $P S$}

In order to compare two shapes A and B whose QSDs have the same number of relevant points (denoted by $n$ ), the similarity between A and B is calculated from (2) as an arithmetic mean of the similarity between relevant points of both shapes cyclically in a clockwise direction. Thus, the calculation of the similarity can start each time at a different relevant point of any of the shapes. When all the possible similarities between relevant points are obtained, the similarity between A and $B$ is the higher value of all of them.

Let us see an example for two triangles, T1 and T2. If T1 $=\left\{P_{1}(0), P_{1}(1), P_{1}(2)\right\}$ and $\mathrm{T} 2=\left\{P_{2}(0), P_{2}(1), P_{2}(2)\right\}$, then three similarities are considered (for simplifying, we denote $\operatorname{Sim}\left(P_{1}(i), P_{2}(j)\right)$ as $\left.\operatorname{Sim}(i, j)\right)$ :

$$
\begin{aligned}
& \operatorname{Sim}_{1}(T 1, T 2)=\frac{1}{3}(\operatorname{Sim}(0,0)+\operatorname{Sim}(1,1)+\operatorname{Sim}(2,2)) \\
& \operatorname{Sim}_{2}(T 1, T 2)=\frac{1}{3}(\operatorname{Sim}(1,0)+\operatorname{Sim}(2,1)+\operatorname{Sim}(0,2)) \\
& \operatorname{Sim}_{3}(T 1, T 2)=\frac{1}{3}(\operatorname{Sim}(2,0)+\operatorname{Sim}(0,1)+\operatorname{Sim}(1,2))
\end{aligned}
$$

and, the final similarity between $\mathrm{T} 1$ and $\mathrm{T} 2$ is the maximum of these three. Note that a correspondence between relevant points of two shapes is provided with this similarity: if the final similarity between the triangles $\mathrm{T} 1$ and $\mathrm{T} 2$ is given from the $\mathrm{Sim}_{2}(\mathrm{~T} 1, \mathrm{~T} 2)$, then the correspondence obtained is: $P_{1}(1) \rightarrow P_{2}(0), P_{1}(2) \rightarrow P_{2}(1)$, and $P_{1}(0) \rightarrow P_{2}(2)$.

On the other hand, if two shapes A and B whose QSDs have a different number of relevant points are compared, then there are some relevant points of one shape with no corresponding points in the other shape. In this case, the points with no corresponding pairs in the other shape are compared with a new relevant point, the void point, and the similarity between both points is zero. Let us suppose that the number of relevant points of the shapes A and B are $n$ and $m$ respectively, and without loss of generality that $n \geq m$. In this case, $n-m$ relevant points of A are compared with the void point, and the rest are compared with the relevant points of B in the same way as in the previous case. 
Thus, the similarity for each one of all possible correspondences between the points of A and $\mathrm{B}$ is obtained as:

$$
\operatorname{Sim}(A, B)=\frac{1}{n} \sum_{P_{A} \in A, P_{B} \in B}^{m} \operatorname{Sim}\left(P_{A}, P_{B}\right)
$$

From here, the final similarity between the shapes A and B, called $\operatorname{Sim} Q S D(A, B)$, is the maximum value of these similarities, that is,

$$
\operatorname{SimQS} D(A, B)=\max _{C}(\operatorname{Sim}(A, B))
$$

where $C$ denotes the set of all possible correspondences between relevant points of A and B.

\subsection{Similarity between $Q C D s$}

The colour names $(Q C)$ are defined from three intervals $I^{H} \times I^{S} \times I^{L}$ (an hypercube) in $\mathfrak{R}^{3}$. Thus, an interval distance between colour names can be defined. Given two hypercubes $H_{1}$ and $H_{2}$ in $\mathfrak{R}^{3}$ with $H_{i}=B_{r_{i}^{H}}\left(c_{i}^{H}\right) \times B_{r_{i}^{S}}\left(c_{i}^{S}\right) \times B_{r_{i}^{L}}\left(c_{i}^{L}\right)$ for $i=1,2$, a distance between colour names is given by:

$$
d_{Q C}\left(H_{1}, H_{2}\right)=\sqrt{\sum_{i=\{H, S, L\}}\left(\left(c_{2}^{i}-c_{1}^{i}\right)^{2}+\left(r_{2}^{i}-r_{1}^{i}\right)^{2}\right)}
$$

Hence, given two colour names in way of hypercubes $Q C_{A}$ and $Q C_{B}$, a similarity $\operatorname{Sim} Q C D$ between them is defined as:

$$
\operatorname{Sim} Q C D\left(Q C_{A}, Q C_{B}\right)=1-\frac{d_{Q C}\left(Q C_{A}, Q C_{B}\right)}{\max \left(d_{Q C}\right)}
$$

where $\max \left(d_{Q C}\right)$ denotes the maximum dissimilarity for all colours defined by $\mathrm{QC}_{L A B 1 . .5}$. Hence, by dividing $d_{Q C}\left(Q C_{A}, Q C_{B}\right)$ and $\max \left(d_{Q C}\right)$ the proportion of dissimilarity related to qualitative colours $\mathrm{QC}_{A}$ and $\mathrm{QC}_{B}$ is obtained, which has values between 0 and 1. Finally, this value is subtracted from 1 with the aim of providing a similarity instead of a dissimilarity.

\subsection{Similarity of $Q Z D s$}

As the features of shape A, TC and L, the qualitative feature Size is also defined as a discretization of values in the reference system SRS. Therefore, applying 1 to $\mathrm{S}_{I N T}$ a matrix of dissimilarity values between the qualitative names in $\mathrm{S}_{L A B}$ can be obtained.

Hence, given two qualitative size names $Q Z_{A}$ and $Q Z_{B}$, a similarity measure $\operatorname{Sim} Q Z D$ between them is defined as:

$$
\operatorname{Sim} Q Z D\left(Q Z_{A}, Q Z_{B}\right)=1-\frac{d_{Q Z}\left(Q Z_{A}, Q Z_{B}\right)}{\max \left(d_{Q Z}\right)}
$$


where $\max \left(d_{Q Z}\right)$ denotes the maximum dissimilarity for all sizes defined by $\mathrm{S}_{L A B}$. Hence, by dividing $d_{Q Z}\left(Q Z_{A}, Q Z_{B}\right)$ and $\max \left(d_{Q Z}\right)$ the proportion of dissimilarity related to qualitative sizes $\mathrm{QC}_{A}$ and $\mathrm{QC}_{B}$ is obtained, which has values between 0 and 1. Finally, this value is subtracted from 1 with the aim of providing a similarity instead of a dissimilarity.

\subsection{Similarity of Object Descriptions}

Given two objects $A \rightarrow\left\{Q S D_{A}, Q C_{A}, Q Z D_{A}\right\}$ and $\mathrm{B} \rightarrow\left\{Q S D_{B}, Q C_{B}, Q Z D_{B}\right\}$ described qualitatively as previously explained, a similarity measure $\operatorname{Sim}(A, B)$ between them can be defined as a weighted-mean of the $\operatorname{Sim} Q S D\left(Q S D_{A}, Q S D_{B}\right), \operatorname{Sim} Q C D\left(Q C_{A}, Q C_{B}\right)$ and $\operatorname{Sim} Q Z D\left(Q Z_{A}, Q Z_{B}\right)$.

\section{Experimentation: Mosaic Assembling}

In this section, the testing scenario is drawn (Section 4.1), the approximate matching algorithm is described (Section 4.2) and the results are explained (Section 4.3).

\subsection{Scenario}

In the scenario of experimentation, some tiles are placed on a working table above which an industrial camera is located as Figure 2 (a) shows. The input of our approach is an image of the mosaic design (Figure 2 (b) shows an example) and an image of the pieces on the table taken by the camera (Figure 2 (c) shows an example). The output of this approach is the corresponding

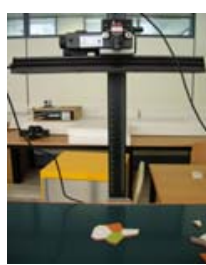

(a) Scenario

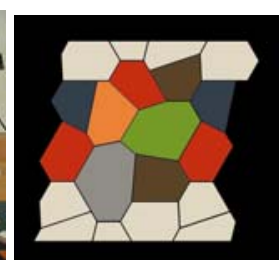

(b) Mosaic design

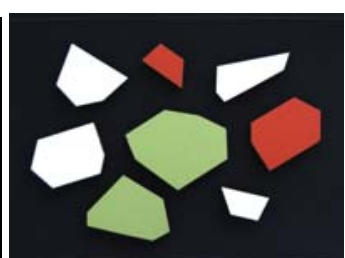

(c) Image captured

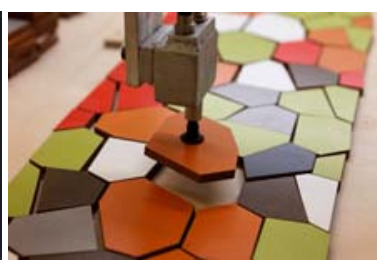

(d) Assembling task

Figure 2: Scenario for assembling tile mosaics by approximate matching: (a) working table and industrial camera; (b) mosaic design; (c) example of a digital image captured by the camera; (d) pick-and-place task

position of each tile in the mosaic. The vision system is composed of two AVT-Guppy F033C cameras, the robot arm used is an IRB 140 ABB robot with 6 degrees of freedom and the pickand-place task is carried out exactly as described by Museros et al. [12] (Figure 2 (d)). 


\subsection{The Approximate Matching Algorithm}

The algorithm for approximate matching that identifies the correspondences of the real tile pieces in the mosaic is detailed in Algorithm 1 and described next. A comparison of qualitative descriptions is carried out for all the possible pairs of objects: an object appearing on the table and an object located on the design which has not been already matched. This comparison is applied in three stages:

1. if the two objects are similar in size ( $\operatorname{SimQZD}\left(O b j_{\text {table }}, O b j_{\text {design }}\right) \geq$ SizeThreshold $)$ the matching algorithm continues (Step 2), otherwise, the location in the design is discarded because the tile is too big or too small for the left space;

2. if the two objects are similar in shape ( $\operatorname{SimQSD}\left(O b j_{\text {table }}, O b j_{\text {design }}\right) \geq S$ hapeThreshold $)$, then the matching algorithm continues (Step 3), otherwise, the location in the design is discarded because its shape is too different from the left space in the mosaic;

3. if the two objects are similar enough in colour $\left(\operatorname{Sim}_{\mathrm{A}} \mathrm{CD}\left(\mathrm{Obj}_{\mathrm{table}}, O \mathrm{Obj}_{\text {design }}\right) \geq\right.$ ColourThreshold $)$, the location in the design is discarded because the colour of the tile does not correspond to the place in the design. Otherwise, that is, the two objects are similar in size, shape and colour, the matching is carried out and the robot picks the tile on the table and places it in the corresponding location in the design.

Finally, if a candidate tesserae does not match to any location in the design, it is discarded.

\subsection{Results}

After testing this approach using different thresholds, successful results are obtained as Figure 3 shows. The input is an image of the mosaic design (Figure 3 (a)) and tests have been carried out on some images of the pieces on the table taken by the camera (Figure 3 (b) and (c)). The closed boundary of the objects within these images is extracted using the segmentation method by Canny [35]. From that boundary, the relevant points of shape and the centroid of each object are extracted, which are then used for obtaining the qualitative descriptions of shape, colour and size of each object. The qualitative descriptions of the objects on the table are compared to the descriptions of the objects in the mosaic design and the final correspondences of real tile pieces in the final mosaic are calculated. The outputs of our approach are the corresponding position of each tile in the mosaic, as Figure 3 (e)-(f) and (h)-(i) shows. 


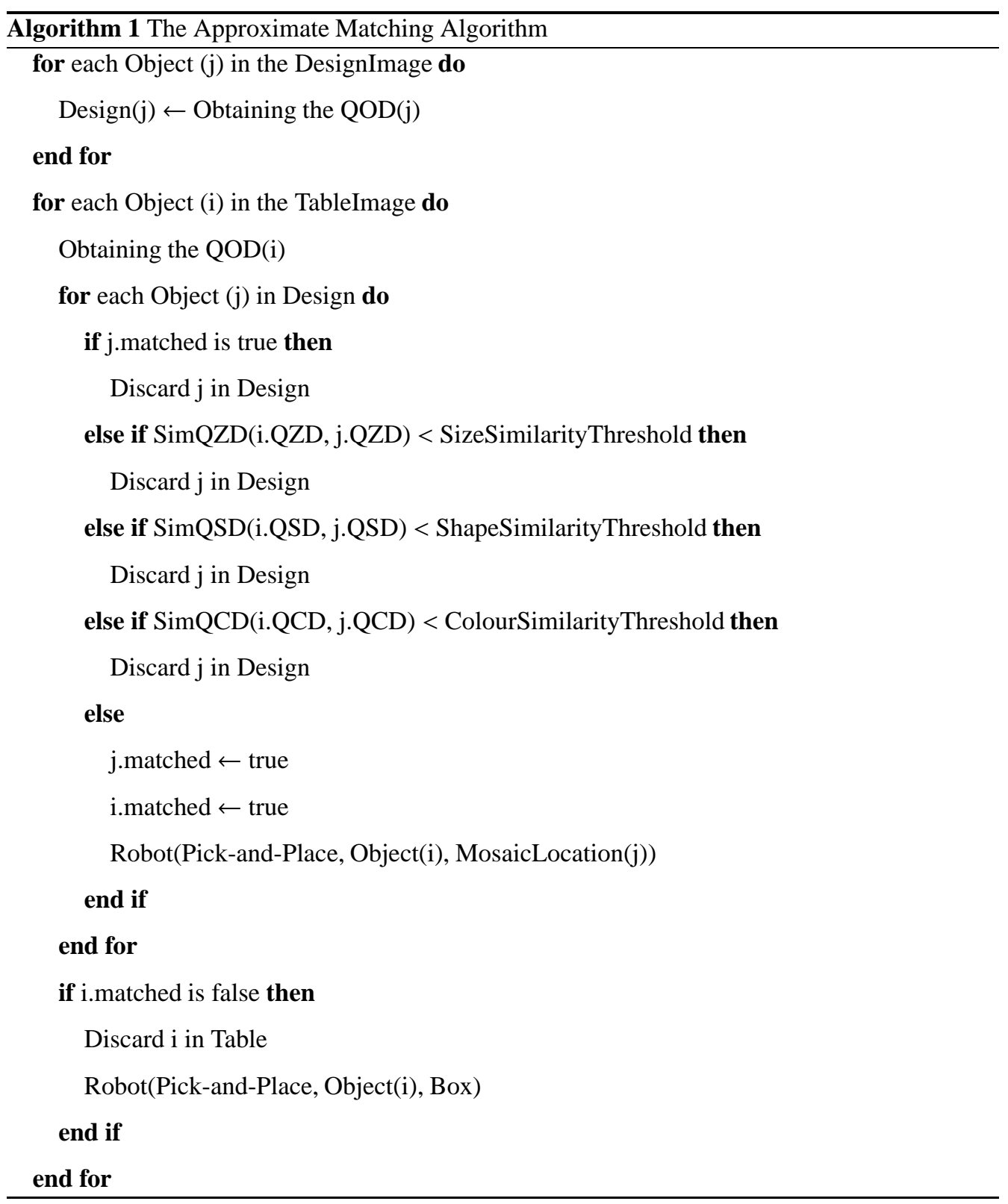




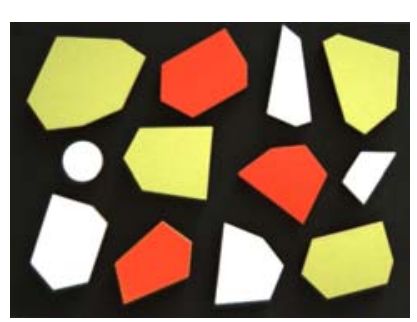

(a) Image-1 captured

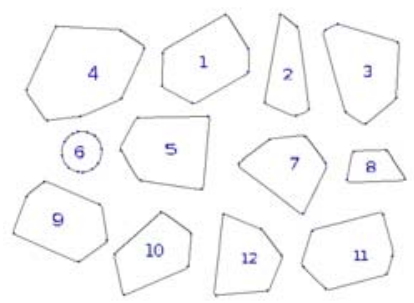

(d) Image-1 processed

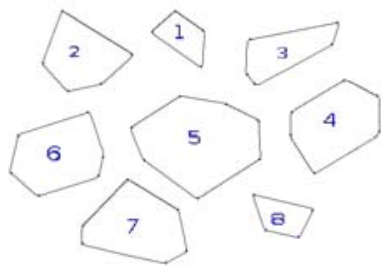

(g) Image-2 processed

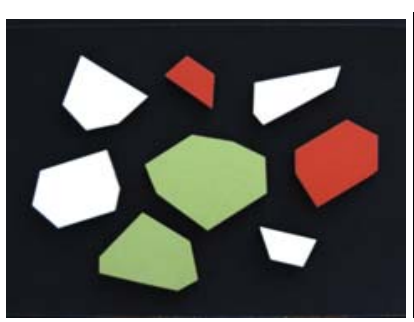

(b) Image- 2 captured

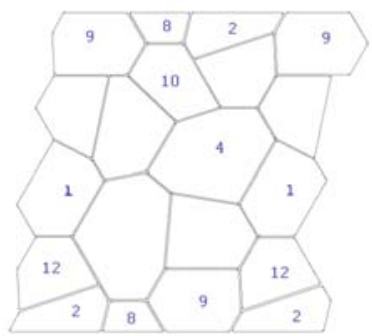

(e) Results if $\operatorname{Sim} Q C D>0.9, \operatorname{Sim} Q S D>$

0.98 and $\operatorname{Sim} Q Z D>0.99$

f) Results if $\operatorname{Sim} Q C D>0.75$, $\operatorname{Sim} Q S D>$

0.98 and $\operatorname{Sim} Q Z D>0.99$

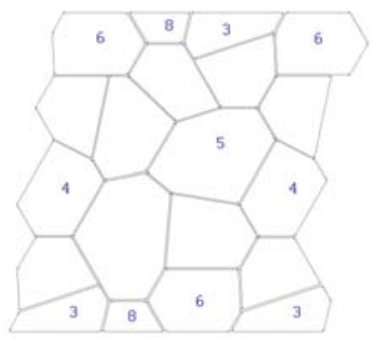

(h) Results if $\operatorname{Sim} Q C D>0.9, \operatorname{Sim} Q S D>$ (i) Results if $\operatorname{Sim} Q C D>0.75, \operatorname{Sim} Q S D>$

0.98 and $\operatorname{Sim} Q Z D>0.99$

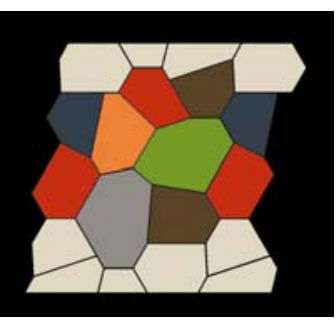

(c) Mosaic design

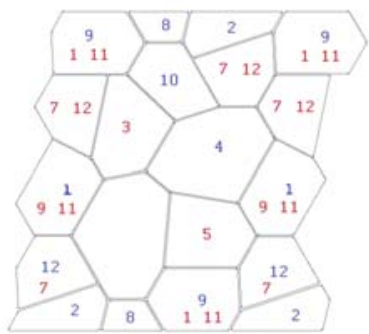

$$
0.98 \text { and Sim } Q Z D>0.99
$$

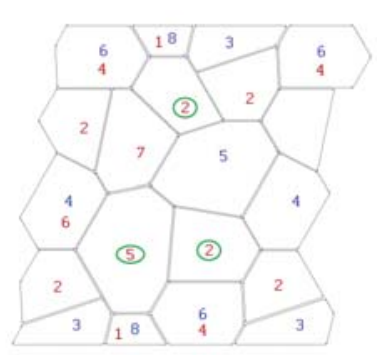

(i) Results if $\operatorname{Sim} Q C D>0$
0.95 and $\operatorname{Sim} Q Z D>0.99$

Figure 3: Scenario for assembling tile mosaics: (a) and (b) digital images captured by the camera; (c) mosaic design; (d) and (g) digital images processed by our approach; and (e), (f), (h) and (i) examples of correspondences found by the approximate matching algorithm.

Figure 3 (f) and (i) shows two examples of the matches obtained with the following similarity thresholds: $\operatorname{Sim} Q Z D \geq 0.99$, $\operatorname{Sim} Q S D \geq 0.98$ and $\operatorname{Sim} Q C D \geq 0.90$. All the matches done in these situations correspond to correct locations in the mosaic design.

Figure 3 (g) shows the matching results relaxing the colour similarity threshold ( $\operatorname{sim} Q C D>$ 


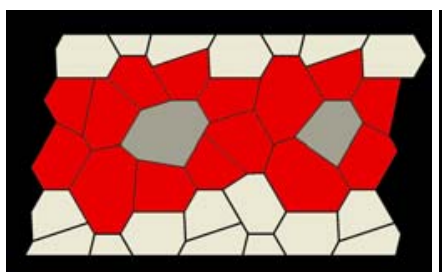

(a)

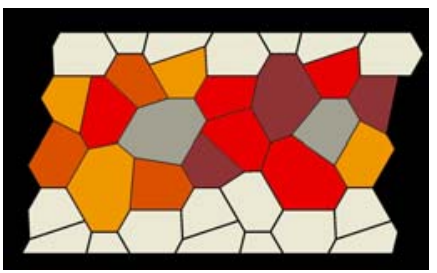

(b)

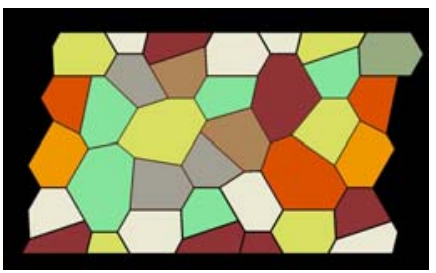

(c)

Figure 4: Results of the application relaxing the colour similarity measure: (a) the initial mosaic design, (b) the mosaic produced by the approximate matching algorithm presented with a $\operatorname{Sim} Q C D \geq 0.75$, and (c) the mosaic produced considering only $\operatorname{Sim} Q Z D \geq 0.99$ and $\operatorname{Sim} Q S D \geq 0.98$.

0.75 , $\operatorname{Sim} Q S D>0.98$ and $\operatorname{Sim} Q Z D>0.99$ ). Note that the numbers in red are the matchings corresponding to the pieces with the same shape and size, but with different colour.

Figure 3 (j) shows the matching results relaxing the colour and shape similarity threshold $(\operatorname{Sim} Q C D>0.75$, $\operatorname{Sim} Q S D>0.98$ and $\operatorname{Sim} Q Z D>0.99)$. In this situation, two aspects are important:

- The first one is that tiles with the same shape and size but different colour (i.e. tiles 2 and 7) are matched in the design.

- The second important aspect is that false positive matches are obtained for tiles 2 and 5 because of the high perceptual similarity of their shapes. However, these false positive matches are not a problem for our approach because they can be controlled by rising the ShapeThreshold.

This approach can generate different colourful mosaics by relaxing the colour similarity threshold, which is a cognitively significant result because the final mosaics obtained are perceived as following a different shape pattern, even if they actually follow the same shape pattern and only colours change, as demonstrated in Figure 4. The mosaic in Figure 4 (b) is generated using a ColourThreshold $=0.75$ and the mosaic in Figure 4 (c) is generated not taking into account the colour of the tile, therefore each tile was assembled in a free place of the mosaic design according to its size, its shape, and the order of appearance on the working table.

Using a computer Intel Core i5 processor at $2.27 \mathrm{GHz}$ and $4 \mathrm{~GB}$ of RAM, running under a Ubuntu 10.04 (lucid) with a Linux kernel 2.6.32-21-generic, the presented approach describes and matches a tesserae in an average time of 0.4 seconds. The robot arm speed is set to $20 \mathrm{~m} / \mathrm{min}$; 
and the distance between the input table and the mosaic assembly table is $1 \mathrm{~m}$. Therefore, the robot system needs an average time of 2 seconds to move from the working table to the mosaic. Taking into account these parameters the system needs 2.4 seconds to pick and place a tile in its corresponding place and orientation in the final mesh, and 2 seconds more to return to the working table. Therefore it needs 4.4 seconds for placing each tile. If all the tesserae match in the design, it will require $7.3 \mathrm{~min}$ to assemble a mosaic of 100 pieces which represents a big improvement on the time taken by the hand assembly method currently used.

\section{Discussion}

In this section, a discussion is given regarding further applications of the presented approach (Section 5.1), a comparison between the results obtained in the literature and the results provided by the presented approach is given (Section 5.2) and, finally, its economic justification is explained (Section 5.3).

\subsection{Other Applications}

Besides automating tile mosaic assembly and producing colourful mosaics, the similarity measures defined in this paper can be used for solving the automation of trencad is assembling. A trencadís mosaic is built by pieces which are not pre-manufactured for the design, but randomly created by tile smashing, and the space between tiles is variable as shown in Figure 5 (a) and (b). As far as we are concerned, there are not works in the literature that have started to tackle this problem.

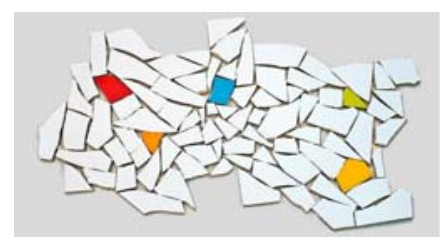

(a) Incomplete Trencadís

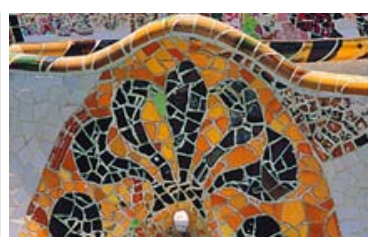

(b) Trencadís

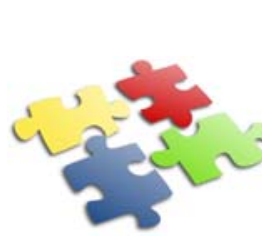

(c) Assembling Jigsaw (d) Complete Jigsaw puzzle

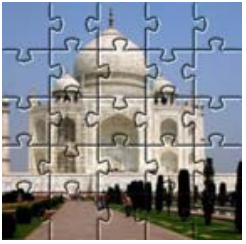

puzzle

Figure 5: Other applications: (a)-(b) Trencadís assembly; (c) Jigsaw puzzle with homogeneously coloured pieces; (d) Jigsaw puzzle with textured pieces.

Another interesting application to which the presented similarity measures can be applied is the Jigsaw puzzle solving with homogeneously coloured pieces (see Figure 5 (c)). The approach 
presented in this paper can qualitatively describe: (i) the characteristic shape of the Jigsaw pieces which have four right angles and some concavities; (ii) their homogeneous colour; and (iii) their size which is nearly always the same for all the pieces (contrary to mosaics). In the literature, Kosiba et al. [36] assemble Jigsaw puzzles taking into account only the shapes of the pieces. The assembly of Jigsaw puzzles that built a real image needs further image processing for identifying the location of the piece in the complete image. However, its solution is unique, in contrast to trencadís or other mosaic assembling. In the literature, Yao and Shao [37] provide a solution to these Jigsaw puzzles using boundary shape matching and image merging. In the future, we intend to solve this problem using qualitative descriptions which are closer to the way human beings describe what they see and explain their reasoning.

Note that the matching algorithm presented in this paper is approximate because allows assembling tiles in the mosaic which are similar to the ones in the design. However, the shape and size similarity threshold must be high because the design is predefined from the beginning and another result is not desired. This also happens when solving a Jigsaw puzzle which defines a photograph. However, when assembling other kind of mosaics, such as trencadís, the matching algorithm will be used more effectively because there will be multiple possibilities of assembling tiles to obtain a final mosaic.

\subsection{Comparison to other Approaches}

In the scenario of the presented approach, there is not a predefined set of recognizable tiles, each mosaic design may be composed by any number of different tiles, which differs from previous works by Museros et al. [9, 10, 11, 12], in which the set of tiles on the table must be those composing the final design.

Besides, the presented approach outperforms the results of the pilot study carried out by Falomir et al. $[13,14]$ justifying the success of introducing the qualitative size parameter by obtaining a more precise matching and more cognitive false positives when relaxing the shape similarity threshold.

Regarding the computational time, note that the approach presented here accelerates Museros' et al. $[9,10,11,12]$ process of pick-and-place a tesserae from 3 seconds to 2.4 seconds, that is 0.6 seconds per tile. For instance, this is a reduction of 1 minute (from $8.3 \mathrm{~min}$ to $7.3 \mathrm{~min}$ ) assembling a mosaic composed of 100 pieces. 
The presented approach also improves the times provided by Rhinoceros system [3], which assembles only squared tesserae with the same size and whose placement rates are 7, 10, and 12 seconds on average per tesserae.

The Tilemyzer system ${ }^{5}$ can produce a $15 \times 15$ mosaic grid ( 225 pieces) in 3 minutes, which is an average time of 0.8 seconds for placing a tesserae. This system avoids the picking time process because uses cartridges containing squared tesserae with the same colour and size. However, although it is a system faster than the approach presented here, it cannot produce mosaics containing tesserae of different shapes, sizes and orientations.

\subsection{Economic Justification of the Approach}

Since mosaics in decorative strips or borders using not squared tesserae are hand assembled nowadays, their manufacturing costs and time to market are high and therefore their competitiveness in the market its lower. In order to introduce the automated system presented in this paper in a mosaic factory, an invest in a PC, a robot arm, two industrial cameras and two tables is needed. The most expensive piece of infrastructure is the robot arm which can be used in other applications, such as painting tiles. Therefore, the cost of the investment is low in comparison to the benefits that a company may receive from this system. These may include the capability to react quickly to changing markets, with innovative products, a good on-time delivery service and a competitive cost in order to offer good prices, which determines the competitiveness of the manufacturing industry, such as the ceramic, glass and natural industrial sectors, mainly made up of SMEs. In fact, products with specific features and high quality, such as mosaics, often have the best market opportunities. Mosaics represent a high-added value in wall and floor ceramics, glass and natural stone covering products.

Therefore, the economic benefits for the individual SMEs in the ceramic sector are: (i) cost reduction of more than $35 \%$, leading to greater competitiveness; (ii) an increase in the product offer by commercializing new and highly valueadded products with competitive prices: mosaics, which will enable them to increase sales; and (iii) greater flexibility in order to respond rapidly to market demands and customer requirements.

\footnotetext{
${ }^{5}$ Tilemyzer: http: //www.tilemyzer.com (Access date: Oct 2012)
} 


\section{Conclusions and Future Work}

A computational approach that describes qualitatively the shape, colour and size of objects within digital images is presented. Three similarity measures for comparing these qualitative descriptions are defined based on conceptual neighbourhood diagrams and interval distances. And a similarity measure between the qualitative description of two objects is given.

Our approach is not tied to a specific application. It is a knowledge-representation method that can be applied to the problem of quantifying the similarity of any pair of objects in digital images. However, the problem of mosaic assembly is an industrially relevant scenario that showcases our results.

Therefore, the application of assembling tile mosaics has been used in order to test that: (i) the models for qualitative description of the shape, colour and size of real tile objects extract all the relevant information needed in the task; and (ii) the measures of similarity defined using interval distances in $\mathfrak{R}$ and $\mathfrak{R}^{3}$ are effective for the presented case of study.

Finally, a remarkable result of the current approach is that it can accelerate the tile matching process and it can produce perceptually visual different mosaics from a given design just by relaxing the colour similarity threshold.

As future work, we intend to: (i) define a similarity measure between object locations for comparing tesserae that contain holes or other objects inside; and (ii) apply the similarity measures defined in this paper to the automation of trencadis mosaic assembling and Jigsaw puzzle solving.

\section{Acknowledgements}

This work has been partially supported by Universitat Jaume I (Fons del Pla Estratégic de 2011/2012), by the Zentrale Forschungsförderung der Universität Bremen under the project name "Providing human-understandable qualitative and semantic descriptions", and by the Spanish Ministry of Science and Innovation under project ARTEMISA (TIN2009-14378-C02-01).

\section{References}

[1] C. Chailloux, J.-M. L. Caillec, D. Guriot, B. Zerr, Intensity-based block matching algorithm for mosaicing sonar images, IEEE Journal of Oceanic Engineering 36 (4) (2011) 627-645. 
[2] J. Liang, D. DeMenthon, D. Doermann, Camera-based document image mosaicing, in: Proceedings of the 18th International Conference on Pattern Recognition - Volume 02, ICPR '06, IEEE Computer Society, Washington, DC, USA, 2006, pp. 476-479.

[3] M. Bechthold, A. Kane, J. King, P. Michalatos, Robotic tile placement: Tools, techniques, and feasibility, Gerontechnology 11 (2).

[4] R. Navon, Process and quality control with a video camera, for a floor-tilling robot, Automation in Construction 10 (2000) 113-125.

[5] A. Oral, F. Erzincanli, Computer-assisted robotic tiling of mosaics, Robotica 22 (2) (2004) 235-239.

[6] B. Kaya, A. Berkay, F. Erzincanli, Robot assisted tiling of glass mosaics with image processing, Industrial Robot: An International Journal 32 (5) (2005) 388-392.

[7] I. Cayiroglu, B.-E. Demir, Computer assisted glass mosaic tiling automation, Robot. Comput.-Integr. Manuf. 28 (5) (2012) 583-591.

[8] A. Oral, E.-P. Inal, Marble mosaic tiling automation with a four degrees of freedom cartesian robot, Robot. Comput.-Integr. Manuf. 25 (3) (2009) 589-596.

[9] L. Museros, M. T. Escrig, A qualitative theory for shape representation and matching for design, in: European Conference on Artificial Intelligence (ECAI), 2004, pp. 858-862.

[10] L. Museros, Qualitative theories on shape representation and movement. application to industrial manufacturing and robotics, Ph.D. thesis, Departament Ciències de la Computació i Intel.ligència Artificial, Universitat Jaume I, E-12071, Castellón, SPAIN, ISBN: 978-84-691-6583-6 URL: http://www.tdx.cat/TDX-0909108-142621/ (2006).

[11] L. Museros, M. T. Escrig, Automating assembly of ceramic mosaics using qualitative shape matching, in: The 2007 IEEE/RSJ International Conference on Intelligent Robots and Systems, ISBN: 1-4244-0912-8, 2007.

[12] L. Museros, Z. Falomir, F. Velasco, L. Gonzalez-Abril, I. Martí, 2D qualitative shape matching applied to ceramic mosaics assembling, Journal of Intelligent Manufacturing 22 (2011) 1-11.

[13] Z. Falomir, I. Martí, W. Viana, L. Museros, M. T. Escrig, A pragmatic approach for qualitative shape and qualitative colour similarity matching, in: R. A. et al. (Ed.), Artificial Intelligence Research and Development, Vol. 220 of Frontiers in Artificial Intelligence and Applications, IOS Press, Amsterdam, 2010, pp. 281-290.

[14] Z. Falomir, Qualitative Distances and Qualitative Description of Images for Indoor Scene Description and Recognition in Robotics, Universitat Jaume I at Castelló (Spain) and Universität Bremen (Germany), ISBN 978-84-6953694-0, 2011.

[15] B. J. Super, Fast correspondence-based system for shape retrieval, Pattern Recogn. Lett. 25 (2) (2004) 217-225.

[16] H. Ling, D. W. Jacobs, Shape classification using the inner-distance, IEEE Transactions on Pattern Analysis and Machine Intelligence 29 (2007) 286-299.

[17] X. Bai, X. Yang, L. Latecki, Detection and recognition of contour parts based on shape similarity, Pattern Recogn. 41 (7) (2008) 2189-2199.

[18] G. Mori, S. Belongie, J. Malik, Shape contexts enable efficient retrieval of similar shapes, in: CVPR, IEEE Computer Society, 2001, pp. 723-730.

[19] T. B. Sebastian, P. N. Klein, B. B. Kimia, Shock-based indexing into large shape databases, in: 7th European Conference on Computer Vision (ECCV), Springer-Verlag, London, UK, 2002, pp. 731-746.

[20] D. Macrini, K. Siddiqi, S. J. Dickinson, From skeletons to bone graphs: Medial abstraction for object recognition, 
in: CVPR, IEEE Computer Society, 2008.

[21] B. Gottfried, Qualitative similarity measures: The case of two-dimensional outlines, Computer Vision and Image Understanding 110 (1) (2008) 117-133.

[22] Z. Falomir, J. Almazán, J. Grande, L. Museros, M. T. Escrig, A similarity calculus for comparing qualitative shape descriptions, in: S. S. et al. (Ed.), Artificial Intelligence Research and Development, Vol. 202 of Frontiers in Artificial Intelligence and Applications, IOS Press, Amsterdam, 2009, pp. 318-326.

[23] B. Kuijpers, B. Moelans, N. V. D. Weghe, Qualitative polyline similarity testing with applications to query-bysketch, indexing and classification, in: R. de By, S. Nittel (Eds.), 14th ACM-GIS, ACM, 2006, pp. 11-18.

[24] A. Schuldt, B. Gottfried, O. Herzog, Retrieving shapes efficiently by a qualitative shape descriptor: The scope histogram, in: Image and Video Retrieval, 5th International Conference, 2006, pp. 261-270.

[25] M. Sarifuddin, R. Missaoui, A new perceptually uniform color space with associated color similarity measure for contentbased image and video retrieval, in: Multimedia Information Retrieval Workshop, 28th annual ACM SIGIR conference, 2005, pp. 3-7.

[26] K. N. Plataniotis, A. N. Venetsanopoulos, Color image processing and applications, Springer-Verlag, NY, USA, 2000.

[27] M. Seaborn, L. Hepplewhite, T. J. Stonham, Fuzzy colour category map for the measurement of colour similarity and dissimilarity, Pattern Recognition 38 (2) (2005) 165-177.

[28] L. D. Griffin, Similarity of psychological and physical colour space shown by symmetry analysis, Color Research and Application 26 (2) (2001) 151-157.

[29] Z. Falomir, J. Almazán, L. Museros, M. T. Escrig, Describing 2D objects by using qualitative models of color and shape at a fine level of granularity, in: Proc. of the Spatial and Temporal Reasoning Workshop at the 23rd AAAI Conference on Artificial Intelligence, ISBN: 978-1-57735-379-9, 2008.

[30] Z. Falomir, L. Museros, L. Gonzalez-Abril, M. T. Escrig, J. A. Ortega, A model for qualitative description of images based on visual and spatial features, Computer Vision and Image Understanding 116 (2012) 698-714.

[31] C. Steger, On the calculation of arbitrary moments of polygons, Tech. Rep. FGBV-96-05, Technische Universität München (1996).

[32] W. Cochran, Sampling techniques, 3rd edition. New York: Wiley and Sons., 1977.

[33] C. Freksa, Conceptual neighborhood and its role in temporal and spatial reasoning, in: M. G. Singh, L. TravéMassuyès (Eds.), IMACS Workshop on Decision Support Systems and Qualitative Reasoning, North-Holland, Amsterdam, 1991, pp. 181-187.

[34] L. Gonzalez-Abril, F. Velasco, C. Angulo, J. A. Ortega, F. Ruiz, Sobre núcleos, distancias y similitudes entre intervalos, Inteligencia Artificial. Revista Iberoamericana de IA (23) (2004) 111-117.

[35] J. F. Canny, A computational approach to edge detection, IEEE Transactions on Pattern Analysis and Machine Intelligence (TPAMI) 8 (1986) 679-697.

[36] D. A. Kosiba, P. M. Devaux, S. Balasubramanian, T. L. Gandhi, K. Kasturi, An automatic jigsaw puzzle solver, in: Conference A: Computer Vision and Image Processing., Proceedings of the 12th IAPR International Conference on Pattern Recognition, Vol. 1, 1994, pp. 616-618.

[37] F.-H. Yao, G.-F. Shao, A shape and image merging technique to solve jigsaw puzzles, Pattern Recognition Letters 24 (12) (2003) 1819-1835. 